Rong $\mathrm{Li}$

Quanzhao Sun $\bowtie$

Jie Zhang

Yanming Song

Guolai Yang

Baochun Lu

https://doi.org/10.21278/TOF.44305

ISSN 1333-1124

eISSN 1849-1391

\title{
MULTIPLE ATTRIBUTE DECISION MAKING WITH INTERVAL UNCERTAINTY FOR ARTILLERY RECOIL RESISTANCE
}

\begin{abstract}
Summary
To reduce loads acting on artillery carriages and obtain better values of recoil resistance, a study on multiple attribute decision making with interval uncertainty of the liquid orifice of a recoil mechanism was conducted. Taking the dimensions of the liquid orifice as the uncertainty variables, the uncertainty optimization model and algorithm based on three parameter interval were used to achieve the optimization schemes of the throttling bar outer dimensions with different tolerance grades. The multiple optimization schemes were sorted by employing the multiple attribute decision making method, in which the attribute weights were determined based on the maximum deviation method. The results show that the optimal design scheme is the one which considers simultaneously the parameter design and tolerance design of the throttling bar outer diameters. The optimal interval of the recoil resistance peaks and the optimal recoil resistance curve with sufficient fullness and flatness were obtained. The study results are beneficial for artillery design and evaluation concerning both the manufacturability of artillery and particular requirements of recoil resistance.
\end{abstract}

Key words: $\quad$ Artillery recoil resistance, multiple attribute decision making, interval uncertainty, optimization evaluation, uncertainty of dimensions

\section{Introduction}

Artillery recoil mechanisms can provide reasonable resistance and significantly reduce backward force acting on carriages. Hydraulic resistance is caused by the high speed flow of fluid pushed through the liquid orifice by a piston. The expected values of recoil resistance will be obtained by a better design of the liquid orifice area, which is the area between the throttling ring and the throttling bar. As explained by [1], to obtain better performance of artillery recoil resistance, the recoil mechanism is generally optimized while maintaining the original counter-recoil mechanism. Zong et al. [2] established a coupling dynamic model of an artillery recoil mechanism considering the effects of the interaction between the artillery recoil and the overall gun motion, and optimized the dimensions of the throttling bar. Zhou et al. [3] chose the agreement between the expected and predesigned recoil force and the recoil 
length as the objective function, and solved the optimization problem of the throttling bar diameters. Song et al. [4] created an optimization model which takes the throttling bar parameters as design variables and reduces the recoil resistance peak as the objective function within a certain range of recoil length; thus, a gentler curve of recoil resistance was obtained. Liu et al. [5] developed an optimization model of a recoil mechanism based on the strength calculation model, the kinetics model of the gun recoil process, and the optimal design method; finally, optimization solutions were achieved by co-simulation. Li et al. [6] created the optimal design of artillery recoil resistance considering the firing stability by using a dynamic optimization theory. Cui et al. [7] performed the artillery structural parameter optimization based on ADAMS and CPSO algorithms. Unfortunately, most of the available models are deterministic models, which neglect the perturbation of material parameters, loads, and dimensions, as well as the effects of parameter uncertainty on the objective performance. The dimensions of liquid orifice are uncertain due to the errors of machining and assembly. The uncertainty affects the values of recoil resistance and recoil length significantly, and it should be taken into account when designing and evaluating optimization programs for artillery recoil mechanisms.

Interval optimization methods are appropriate approaches to solving problems with known upper and lower limits of uncertainty variables. Guha et al. [8] investigated an adaptive control strategy based on neural networks optimization that can be used for designing controllers for continuous process control problems based on interval analysis. Jiang et al. [9] defined a dimensionless design tolerance index to describe the overall uncertainty of all design variables, and presented an interval optimization model considering the manufacturing errors of design variables, where the possibility degree of interval was used to represent the reliability of the constraints under uncertainty. However, in the optimization stage, there are many results instead of an optimal result. The purpose of evaluation is to determine the best scheme among the non-inferior solutions, and to provide guides for decision makers. Multiple attribute decision making methods are optional in the artillery design optimization and evaluation.

To reduce loads acting on artillery carriages and to obtain better values of recoil resistance, research on multiple attribute decision making with the interval parameter uncertainty of a recoil mechanism is carried out from the point of view of dimensional deviations of liquid orifice, on the basis of our preliminary work [10] concerning the interval uncertainty optimization of artillery recoil resistance. The rest of the paper is organized as follows: in Section 2, a brief introduction into artillery recoil resistance generated by a throttling bar recoil mechanism is given, as well as the uncertainty analysis of the artillery recoil resistance. In Section 3, an interval uncertainty optimization method developed earlier is employed to optimize the recoil resistance and to provide the schemes for evaluation. In Section 4, the principles for establishing an evaluation index system of optimization are presented, and a two-level decision making model is established. The multiple attribute decision making with interval uncertainty, using the weight determination method based on maximum deviation is carried out. Finally, the non-inferior solutions are evaluated and the optimal design of the outer diameters of the throttling bar is determined.

\section{Uncertainty analysis of artillery recoil resistance}

\subsection{Artillery recoil resistance}

Throttling bar recoil mechanisms are widely used in modern artillery. The schematic diagram of a throttling bar recoil mechanism is shown in Fig. 1(a), while that of the throttling bar is shown in Fig. 1(b). The geometry parameters are listed as follows: $L$, working length of the recoil mechanism; $D_{T}$, inner diameter of the recoil cylinder; $d_{T}$, outer diameter of the 
recoil-piston rod; $d_{1}$, inner diameter of the chamber in the recoil-piston rod; $d_{p}$, diameter of the throttling ring; $d_{x}$, dimensions of the throttling bar; $D_{1}$, outer diameter of the recoil brake cylinder. All lengths are in millimetres.

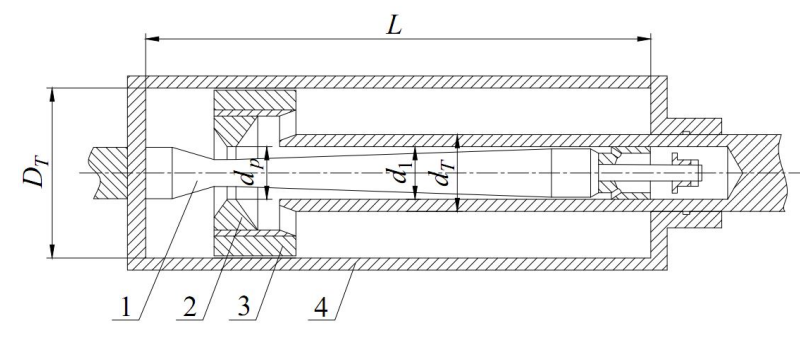

a)

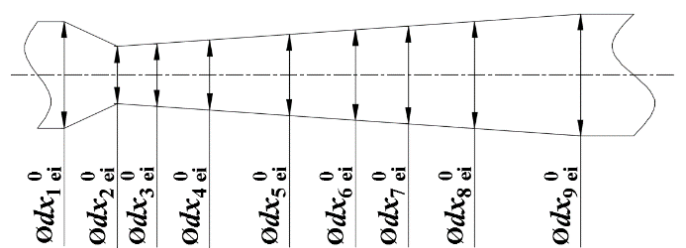

b)

Fig. 1 a) Schematic diagram of the throttling bar recoil mechanism (1- throttling bar, 2- throttling ring, 3- recoilpiston, 4- recoil cylinder); b) Schematic diagram of a throttling bar

The fluid resistance of recoil brake, $F_{\Phi H}$, considering the effects of the clearance between the recoil piston and the recoil brake cylinder, can be expressed as follows:

$$
F_{\Phi H}=\frac{K_{1} \rho}{2}\left[\frac{\left(A_{0}-A_{p}\right)^{3}}{\left(a_{x}+a_{0} \sqrt{K_{1} / K_{1}^{\prime}}\right)^{2}}+\frac{K_{2}}{K_{1}} \frac{A_{f j}^{3}}{A_{1}^{2}}\right] V^{2}
$$

where $A_{0}$ is the working area of the recoil piston, $A_{p}$ is the area of the throttling ring, $A_{f j}$ is the working area of the return throttling device, $A_{1}$ is the minimum cross section area of the side stream, $\rho$ is the mass density of recoil fluid, $K_{1}$ is the hydraulic drag coefficient of the main stream, $K_{2}$ is hydraulic drag coefficient of the side stream, $a_{x}$ is the area of the liquid orifice, $a_{0}$ is the area of the clearance between the recoil piston and recoil brake cylinder, and $K_{1}^{\prime}$ is the hydraulic drag coefficient of the stream through the clearance.

The differential equation of recoil motion is written as

$$
m_{h} \frac{\mathrm{d}^{2} x}{\mathrm{~d} t^{2}}=F_{p t}-F_{R}
$$

where $x$ is the recoil displacement, $F_{p t}$ is the gun tube resulting force, and $F_{R}$ is the artillery recoil resistance, which can be calculated by

$$
F_{R}=F_{\Phi H}+F_{f}+F+F_{T}-m_{h} g \sin \varphi
$$

where $F_{f}$ is the recuperator force, $F$ is the friction force generated by the tightening device, $F_{T}$ is the friction force generated by the cradle guide, $m_{h}$ is the recoiling part mass, and $\varphi$ is the elevating angle.

\subsection{Calculation of uncertainty in recoil resistance}

Manufacturing and assembly are important causes of the dimension uncertainty of the liquid orifice. The diameters of the break points of the throttling bar, $d x_{i}$, the inner diameter of the throttling ring, $d_{p}$, the clearance between the recoil piston and the recoil brake cylinder, $\Delta \delta_{1}$, and the assembly error of the starting position of the throttling ring and the throttling bar in the axial direction, $\Delta l$, were taken as uncertainty variables. The ranges of the variables were determined from the design parameters of the artillery, as listed in Table 1 . The initial values of $d x_{i}$ were their nominal dimensions, the initial value of $\Delta \delta_{1}$ was $0.315 \mathrm{~mm}$, and the initial value of $\Delta l$ was $0 \mathrm{~mm}$. The ranges of the uncertainty variables were: $d_{p} 43_{0}^{+0.039}, \Delta \delta_{1} 0.25 \sim 0.38$, and $\Delta l-10 \sim 10$ (units: $\mathrm{mm}$ ). A total of 500 samples was obtained in the symmetric Latin 
hypercube test design. For each sample, the differential equation of recoil motion was used to simulate the recoil problem under normal shooting conditions. The initial values of the computed recoil resistance peak, $F_{R \max }$, and the recoil length, $\lambda$, were $190982 \mathrm{~N}$ and $865.8 \mathrm{~mm}$, respectively, and their calculated minimum and maximum values are listed in Table 1.

In a traditional deterministic analysis of recoil resistance, the uncertainty of the dimensions of the liquid orifice is not taken into account. At the same time, the traditional optimization cannot simultaneously obtain the optimal structural dimensions and tolerance ranges in the design. For an optimization problem, the perturbation of objects and constraints will lead to poor robustness of optimization results. It is necessary to conduct interval optimization and decision making by considering the dimension uncertainty of the liquid orifice.

\section{Interval uncertainty optimization of artillery recoil resistance}

\subsection{Uncertainty optimization model with three parameter interval}

The uncertainty optimization of recoil resistance was carried out by optimizing the throttling bar outer dimensions, which were the main parameters determining the area of liquid orifice. The dimensions of a throttling bar are usually uncertain and are distributed over the specified tolerance range, due to the errors of machining and assembly. The tolerance range is a region defined by the upper and the lower limit, and it is an interval in mathematical terms. Nominal dimensions are the criterion for selection and the base for deterministic design methods. Tolerances, which depend on the accuracy of machining, affect the manufacturing costs. To obtain better artillery recoil resistance, the interval uncertainty optimization method developed by the authors of this paper [10] was applied to determine the nominal dimensions and tolerances of the throttling bar.

\section{(1) Construction of three parameter interval}

A three parameter interval was constructed based on the nominal dimension and the tolerance level. For an interval $x^{I}=\left[x^{L}, x^{R}\right]$, let $x^{t}=x^{R}-x^{L}$, where $x^{t}$ is the tolerance. The tolerance level of $x^{t}$ can be found in tables of standard tolerance and standard tolerance level contained in standards, which is $x^{\mathrm{TT} n}$. Define $x^{g}$ as the nominal size of the interval; the coefficient $x^{\theta}, x^{\theta}=\left(x^{g}-x^{L}\right) /\left(x^{R}-x^{L}\right)$, describes the position relationship between the nominal size of the interval and the interval tolerance. The three parameter interval is expressed as

$$
x^{I}=\left\langle x^{g}, \quad x^{\mathrm{ITn} n}, x^{\theta}\right\rangle=\left\{x \mid x^{g}-x^{\theta} \cdot x^{t}<x<x^{g}+x^{\theta} \cdot x^{t}, x^{\mathrm{TT} n} \rightarrow x^{t}, x 、 x^{g} 、 x^{\theta} \in R\right\}
$$

(2) Transformation of three parameter interval design variables

The design variables of the three parameter interval were transformed into two parameter intervals by the tolerance constraint equations. The throttling bar was a bar of variable cross-section; it consisted of eight sections. The diameter of the ninth break point, with a form of diagonal tolerance, was transformed into the same form as the first eight points $\left(42.8_{-0.1}^{0}=43_{-0.3}^{-0.2}\right)$. The diameters of the throttling bar were in the basic shaft system, and the coefficient $x^{\theta}$ was equal to zero, $x_{i}^{\theta}=0$. The tolerance levels of the diameters were the same; they were set as $d x_{i}^{\mathrm{IT} n}=\mathrm{IT} n$ in the optimization.

(3) Deterministic transformation of the objective and the constraint function

The deterministic transformation of the objective and the constraint function is essentially the ordering of the augmented objective intervals by using penalty function methods. First, the method based on the possibility degree of interval [11] was used to compare the intervals, and the probability matrix was formed. The sorting formula presented in [12] was used to obtain the order. 
(4) Uncertainty optimization model of three parameter interval

Through the deterministic transformation, a general optimization model can be transformed into the uncertainty optimization model of three parameter interval. The main goal was to obtain the minimum recoil resistance peak in the case of stationary recoil lengths. According to the calculated results of the recoil length provided in Section 2.2, the recoil length was constrained to be less than or equal to $895 \mathrm{~mm}$. To avoid the phenomenon of clamping between the throttling bar root and the throttling ring, the assembly clearance was constrained to be greater than or equal to $\Delta \delta_{2}$. Taking into account the dimension uncertainty of the liquid orifice, a set of values of each uncertainty variable usually results in a set of values for the recoil resistance peak, which can be treated as an interval, i.e. $F_{R \max }^{I}=\left[\left(F_{R \max }\right)_{\max },\left(F_{R \max }\right)_{\min }\right]$. The objective was to minimize the interval of recoil resistance peak, $F_{R \max }^{I}$. The uncertainty optimization model based on the three parameter interval was established; it can be expressed as [10]

$$
\begin{cases}\min & f\left(d x^{I}, d p^{I}, \Delta \delta_{1}^{I}, \Delta l^{I}\right)=F_{R \max }^{I} \\ \text { where } & d x^{I}=\left(\left\langle d x_{1}^{g}, d x_{1}^{\mathrm{IT} n}, d x_{1}^{\theta}\right\rangle, \ldots,\left\langle d x_{9}^{g}, d x_{9}^{\mathrm{IT} n}, d x_{9}^{\theta}\right\rangle\right) \\ \text { s.t } \quad & \lambda^{I}\left(d x^{I}, d p^{I}, \Delta \delta_{1}^{I}, \Delta l^{I}\right) \leq \lambda_{0} \\ & d x_{i \min } \leq d x^{I} \leq d x_{i \max }-\Delta \delta_{2} \\ & d x_{i}^{\mathrm{IT} n}=\mathrm{IT} n, \quad d x_{i}^{\theta}=0, \quad i=1,2, \ldots, 9\end{cases}
$$

where $d x_{i \min }$ and $d x_{i \max }$ are the minimum and the maximum values of the nominal diameters of the throttling bar sections, respectively, and $\lambda^{I}$ is the interval of recoil length with the dimension uncertainty of liquid orifice being considered.

\subsection{Optimization solutions}

An optimization algorithm with a two-layer structure was adopted to solve the above problem. The NSGA-II genetic algorithm was used in the outer layer to find the optimal design vector, while the inner layer based on a symmetric Latin hypercube design [13] was used to calculate the objective function and the constrained function intervals. A flowchart of the algorithm can be found in our previously conducted study [10].

Five cases with tolerance levels of the throttling bar diameters from IT8 to IT12 were solved. The optimized results of recoil resistance peak and recoil length for the five cases are listed in Table 1. To quantitatively evaluate the recoil resistance curves and assess the performance of recoil mechanisms, the index of fullness, $\alpha$, and the index of flatness, $\beta$, were introduced [14]. The higher the value of $\alpha$, the better the fullness. On the other hand, the smaller the value of $\beta$, the better the flatness. The indices of fullness before and after optimization were marked as $\alpha_{1}$ and $\alpha_{2}$, respectively; $\alpha_{1}=0.9323$ and $\alpha_{2}=0.9703$. The indices of flatness before and after optimization were marked as $\beta_{1}$ and $\beta_{2}$, respectively; $\beta_{1}=3.210 \times 10^{5}$ and $\beta_{2}=3.098 \times 10^{5}$. This indicates that the fullness and flatness were both improved after the optimization. The results show that the recoil resistance peaks were reduced, while the recoil resistance curves were gentler and their fullness was better. The recoil resistance was optimized in the case of stationary recoil lengths. The optimal design schemes of the throttling bar outer diameters simultaneously considering the nominal dimensions and the tolerance ranges were achieved. 
Table 1 Optimization results of cases with different tolerance levels [10]

\begin{tabular}{ccccccc}
\hline $\begin{array}{c}\text { Tolerance } \\
\text { levels }\end{array}$ & $\begin{array}{c}\text { IT10 (Before } \\
\text { optimization) }\end{array}$ & IT8 & IT9 & IT10 & IT11 & IT12 \\
\hline$d x_{1} / \mathrm{mm}$ & $36.7_{-0.1}^{0}$ & $38.8_{-0.039}^{0}$ & $38.8_{-0.062}^{0}$ & $38.7_{-0.1}^{0}$ & $38.9_{-0.16}^{0}$ & $38.6_{-0.25}^{0}$ \\
$d x_{2} / \mathrm{mm}$ & $33.4_{-0.1}^{0}$ & $34.4_{-0.039}^{0}$ & $34.5_{-0.062}^{0}$ & $34.6_{-0.1}^{0}$ & $34.6_{-0.16}^{0}$ & $35_{-0.25}^{0}$ \\
$d x_{3} / \mathrm{mm}$ & $35.8_{-0.1}^{0}$ & $35.6_{-0.039}^{0}$ & $35^{0} 7_{-0.062}^{0}$ & $35.8_{-0.1}^{0}$ & $35.8_{-0.16}^{0}$ & $35.8_{-0.25}^{0}$ \\
$d x_{4} / \mathrm{mm}$ & $36.6_{-0.1}^{0}$ & $36.2_{-0.039}^{0}$ & $36.2_{-0.062}^{0}$ & $36.3_{-0.1}^{0}$ & $36.3_{-0.16}^{0}$ & $36.6_{-0.25}^{0}$ \\
$d x_{5} / \mathrm{mm}$ & $38.1_{-0.1}^{0}$ & $37.7_{-0.039}^{0}$ & $37.8_{-0.062}^{0}$ & $37.8_{-0.1}^{0}$ & $37.9_{-0.16}^{0}$ & $37.9_{-0.25}^{0}$ \\
$d x_{6} / \mathrm{mm}$ & $39.1_{-0.1}^{0}$ & $38.6_{-0.039}^{0}$ & $38.6_{-0.062}^{0}$ & $38.7_{-0.1}^{0}$ & $38.7_{-0.16}^{0}$ & $38.7_{-0.25}^{0}$ \\
$d x_{7} / \mathrm{mm}$ & $40.2_{-0.1}^{0}$ & $40_{-0.039}^{0}$ & $40_{-0.062}^{0}$ & $40.1_{-0.1}^{0}$ & $40.2_{-0.16}^{0}$ & $40.5_{-0.25}^{0}$ \\
$d x_{8} / \mathrm{mm}$ & $41.6_{-0.1}^{0}$ & $42_{-0.039}^{0}$ & $42_{-0.062}^{0}$ & $42.2_{-0.1}^{0}$ & $41.9_{-0.16}^{0}$ & $42.55_{-0.25}^{0}$ \\
$d x_{9} / \mathrm{mm}$ & $43_{-0.3}^{-0.2}$ & $42.5_{-0.039}^{0}$ & $42.5_{-0.062}^{0}$ & $42.5_{-0.1}^{0}$ & $42.6_{-0.16}^{0}$ & $42.7_{-0.25}^{0}$ \\
$F_{R \max }^{I} / \mathrm{kN}$ & {$[182.4,197.1]$} & {$[168.9,178.8]$} & {$[169.2,179.6]$} & {$[169.9,182.8]$} & {$[170.4,182.7]$} & {$[174.9,192.5]$} \\
$\lambda^{I} / \mathrm{mm}$ & {$[853.2,889.3]$} & {$[860.7,892.0]$} & {$[861.0,891.3]$} & {$[853.6,886]$} & {$[855.8,893.5]$} & {$[837.9,877.2]$} \\
\hline
\end{tabular}

\section{Multiple attribute decision making}

In the optimization stage, there are many non-inferior solutions instead of an optimal solution. In the evaluation stage, the aim is to choose one or a batch of optimal schemes from many optimization results and provide guides for decision makers. The design of artillery is a dynamic process which includes continuous optimization. To obtain an optimal design of the throttling bar outer diameters, the principles for establishing the evaluation index system of optimization are presented and the optimization method to determine the index weights is studied. The multiple attribute optimization of the throttling bar outer diameters, with the attribute values which include both real numbers and the interval numbers, is investigated by employing the multiple attribute decision making method based on maximum deviation.

\subsection{Two-level decision making scheme}

The process of obtaining an optimal scheme by uncertainty optimization usually has two stages: optimization and decision making. Uncertainty optimization provides a number of non-inferior solutions, and the best solution is generally determined by a decision making process. The scheme set $A=\left\{A_{1}, A_{2}, \ldots, A_{5}\right\}$ corresponds to the optimization results of cases IT8, IT9, ..., IT12 in Table 2 , respectively.

The evaluation index system is an organized whole which combines the evaluating indicators of multiple interactions according to certain structures and levels. The evaluation index system is the basis for carrying out the optimization evaluation and it also relates the optimization methods to preferred objects. In general, to establish an evaluation index system, the following principles should be adhered to [15, 16]: (1) Scientific methods should be employed to determine the levels of evaluating indicators. The compositions of the preferred objects, as well as their characteristics, tasks and functions, together with the relationship among various essential factors have to be analysed objectively. The index names and their meanings together with the calculation methods can be established on the basis of scientific analysis. (2) The evaluation index system must be based on the characteristics of the evaluation object, so that the indices can be formed as an organized whole with hierarchy and internal relations. According to the different characteristics of the indicators, classification analysis and comprehensive induction can be performed to obtain a top-down constitution through step by step refinement and combination. Usually one to four layers are adopted because the complexity of the algorithm grows exponentially with more layers. (3) The indices at the same level should be distinguished and relatively independent. As it is very difficult to achieve full 
independence of indicators in practical problems, the requirement that could be met appears to be that the evaluating indicators are not precisely repeated, which may result in overlapping attributes. (4) The evaluating indicators are not the more the better. The criterion to determine the indices for the evaluation process is based on their significance. Therefore, choosing the main evaluating indicators is helpful in establishing an evaluation index system with the essential characteristics of the evaluation object being considered. A reasonable evaluation index system can be built by following the above principles, and good performance of the evaluation index system is necessary to solve the evaluation problems appropriately.

For the current problem, the decision making scheme can be considered from two aspects, manufacturability and characteristics of artillery recoil resistance. The throttling bar is a multi-taper slender rod; the poor rigidity and variable cross-section problems make it difficult to process high-quality products. Therefore, it is necessary to consider the manufacturability in the design of the throttling bar outer diameters when choosing the scheme resulting in optimal recoil resistance. Tolerance level is one of the most important factors in process planning, and the tolerance levels of the throttling bar diameters, $u_{11}$, should be carefully treated. In order to reduce the loads acting on gun carriages, a smaller recoil resistance peak is required. As indicated in Section 3.2, the index of fullness refers to the degree of actual recoil resistance curve filling the ideal recoil resistance curve, and the index of flatness refers to the changing speed of recoil resistance with regard to the displacement. Better fullness and flatness of the recoil resistance curves usually indicates better performance of recoil mechanisms. As for the recoil resistance, the interval of recoil resistance peak, $u_{21}$, as well as the fullness and flatness of the recoil resistance curves, $u_{22}$ and $u_{23}$, respectively, should be taken into account. Recoil length is also one of the most important indicators. It reflects the movement of the recoil part and indicates that the designated recoil position has been reached. Accordingly, the corresponding recoil lengths of the optimized schemes were located in the vicinity of the designed value. It is difficult to judge the different scenarios from the recoil lengths. Consequently, the decision making was studied from the point of the manufacturability of artillery recoil and its performance resistance, with the recoil length disregarded. As shown in Fig. 2, a two-level decision making scheme can be designed, in which the first-level attribute set is $u_{2}=\left(u_{21}, u_{22}, u_{23}\right)$, and the second-level attribute set is $u=\left(u_{1}, u_{2}\right)$.

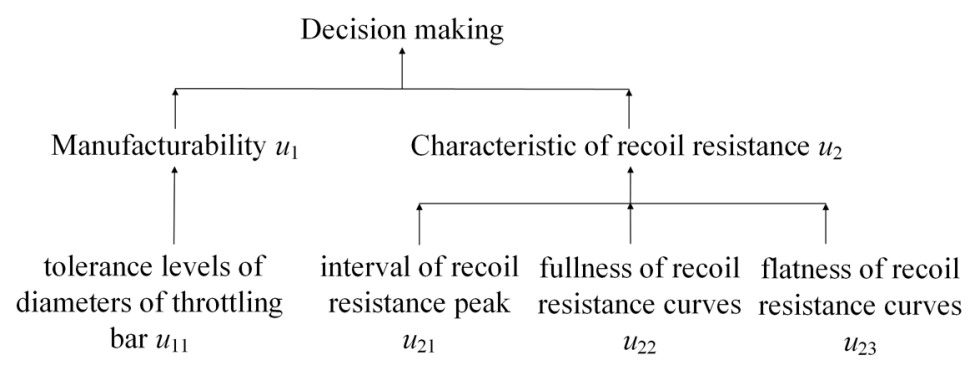

Fig. 2 Two-level decision making scheme

\subsection{Multiple attribute decision making method}

Multiple attribute optimization generally means taking evaluating indicators of the preferred evaluation object as multiple attributes and using multiple attribute decision making methods to optimize the evaluation. Multiple attribute decision making refers to the evaluation and optimization of a limited number of alternatives. The attribute values can be both real numbers and interval numbers. At each level of decision-making system, the attribute set is $\left(u_{1}, u_{2}, \ldots, u_{\mathrm{M}}\right)$. All attribute values assigned to $N$ schemes form the decision matrix of the level. The attribute values can be both interval numbers and real numbers. The multiple attribute decision making method adopted in this paper is as follows: 
(1) Deterministic transformation of attribute values described by interval numbers

The deterministic transformation of the attribute values described by interval numbers is essentially the ordering of the intervals. Similarly, the method based on the possibility degree of interval [11] can be used to compare the intervals, and a probability matrix can be formed. The sorting formula presented in [12] can be used to obtain the order. The possibility degree of interval quantitatively describes the extent to which a range is superior to another. The model is specified as follows:

$$
p\left(A^{I} \leq B^{I}\right)= \begin{cases}0 & , A^{L} \geq B^{R} \\ 0.5 \frac{B^{R}-A^{L}}{A^{R}-A^{L}} \frac{B^{R}-A^{L}}{B^{R}-B^{L}} & , B^{L} \leq A^{L}<B^{R} \leq A^{R} \\ \frac{B^{L}-A^{L}}{A^{R}-A^{L}}+0.5 \frac{B^{R}-B^{L}}{A^{R}-A^{L}} & , A^{L}<B^{L}<B^{R} \leq A^{R} \\ \frac{B^{L}-A^{L}}{A^{R}-A^{L}}+\frac{A^{R}-B^{L}}{A^{R}-A^{L}} \frac{B^{R}-A^{R}}{B^{R}-B^{L}} & \\ +0.5 \frac{A^{R}-B^{L}}{A^{R}-A^{L}} \frac{A^{R}-B^{L}}{B^{R}-B^{L}} & , A^{L}<B^{L} \leq A^{R}<B^{R} \\ \frac{B^{R}-A^{R}}{B^{R}-B^{L}}+0.5 \frac{A^{R}-A^{L}}{B^{R}-B^{L}} & , B^{L} \leq A^{L}<A^{R}<B^{R} \\ 1 \quad & , A^{R}<B^{L}\end{cases}
$$

where $A^{I}$ and $B^{I}$ are the intervals, $A^{R}$ an $A^{L}$ are the upper and lower limits of $A^{I}$, respectively, $B^{R}$ and $B^{L}$ are the upper and lower limits of $B^{I}$, respectively. The possible position relationship between intervals $A^{I}$ and $B^{I}$ is shown in Fig. 3.

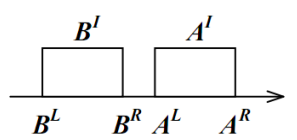

(1)

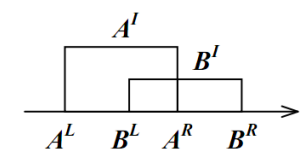

(4)

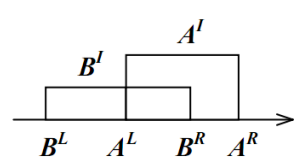

(2)

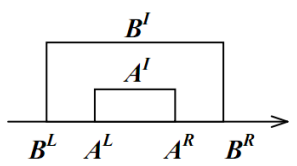

(5)

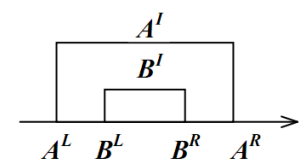

(3)

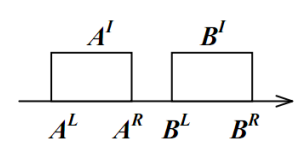

(6)

Fig. 3 Position relationship between intervals $A^{I}$ and $B^{I}$

For a set of attribute values described by interval numbers $q_{i}^{I}=\left[q_{i}^{L}, q_{i}^{R}\right], i=1,2, \ldots, N$, compared with each other by equation (6), one obtains the possibility degree of the interval $p\left(q_{i}^{I} \leq q_{j}^{I}\right)$, which is abbreviated as $p_{i j}$. Then, we can get the probability matrix $\boldsymbol{P}=\left(p_{i j}\right)_{N \times N}$, and calculate the sort vector, $\boldsymbol{\omega}=\left(\omega_{1}, \omega_{2}, \ldots, \omega_{N}\right)^{\mathrm{T}}$, by the formula presented in [12],

$$
\omega_{i}=\frac{1}{N(N-1)}\left[\sum_{j=1}^{N} p_{i j}+\frac{N}{2}-1\right] \quad i=1,2, \ldots, N
$$

which describes the order of the augmented objective interval $q_{i}^{I}$. 
Transform the attribute values of interval numbers to sort the vector $\boldsymbol{\omega}=\left(\omega_{1}, \omega_{2}, \ldots, \omega_{n}\right)^{\mathrm{T}}$ by equations (6) and (7). A new decision matrix $\boldsymbol{Y}$ is obtained by substituting the elements of the sorted vector for the attribute values of the interval numbers, $\boldsymbol{Y}=\left(y_{i j}\right)_{N \times M}$.

(2) Normalization of decision matrix

Attributes can be divided into two types, the cost type (the smaller the better) and the benefit type (the larger the better), according to the characteristics of the property.

The flatness of the recoil resistance curves belongs to the cost type. The normalized attribute values $r_{i j}(i=1,2, \ldots, N, j=1,2, \ldots, M)$ are calculated by

$$
r_{i j}=\frac{\max \left\{y_{1 j}, y_{2 j}, \ldots, y_{N j}\right\}-y_{i j}}{\max \left\{y_{1 j}, \ldots, y_{N j}\right\}-\min \left\{y_{1 j}, \ldots, y_{N j}\right\}}
$$

where $y_{i j}$ is the attribute value.

The benefit type includes the elements of the sort vector transformed from intervals of recoil resistance peaks, the index of fullness of the recoil resistance curves, and the tolerance levels of the throttling bar outer diameters. Their normalized attribute values $r_{i j}$ are calculated by

$$
r_{i j}=\frac{y_{i j}-\min \left\{y_{1 j}, y_{2 j}, \ldots, y_{N j}\right\}}{\max \left\{y_{1 j}, \ldots, y_{N j}\right\}-\min \left\{y_{1 j}, \ldots, y_{N j}\right\}}
$$

(3) Determination of the weight vector, $\boldsymbol{w}=\left(w_{1}, w_{2}, \ldots, w_{M}\right)$

Determination of the index weight is the key to the optimization evaluation system. It is of great importance to determine the weight vector reasonably, especially if there is little difference in the optimization results; in that case, the evaluation value is particularly sensitive to the weight. There are many weight determination methods, evolved from the source of the original data. A suitable weight determination method based on the optimization evaluation problem itself should be considered to avoid an inadequate scheme.

(4) Calculation of the comprehensive attribute values, $z_{i}$, after normalization

$$
z_{i}=\sum_{j=1}^{M} w_{j} r_{i j} \quad i=1,2, \ldots, N, \quad j=1,2, \ldots, M
$$

(5) Arranging the comprehensive attribute values, $z_{i}$

The greater the $z_{i}$, the better the scheme.

\subsection{Weight determination method based on maximum deviation}

The index weight reflects the role and significance of each evaluating indicator in the evaluation index system used in optimization. Reasonable determination of index weights is of great importance for the optimization evaluation. According to the source of original data used for the calculation of index weights, the determination methods of index weights are roughly divided into subjective and objective methods, as well as into some combined methods $[17,18]$. A subjective weight method gives corresponding index weights estimated by decision makers or experts based on their subjective experience and information on each index or scheme. The shortcomings are that these methods are inevitably interfered by human factors. An objective weight method uses the objective information of evaluation objects to determine corresponding index weights, according to the information provided by each index. 
Thus, the interference of human factors when determining the weights is avoided. This method usually uses relatively comprehensive mathematical theories and methods to exclude the source of subjective weights, keeping the weights objective. For a specific optimization evaluation problem, an appropriate weight determination method should be adopted according to the characteristics of the problem.

The schemes of artillery structural tolerance are studied in this paper, and it is clear that the parameters are similar. The deviation caused by the corresponding index may not be too serious. It is not because of the index selection problem, but it is the result of the characteristics of schemes. A subjective method may lead to slight differences between the optimal evaluation values of each scheme, and the optimization results may be particularly sensitive to index weights [19]. Herein, an objective weight method is used to determine the weight of the evaluation indices. The weight determination method based on maximum deviation was adopted to get the attribute weight.

In general, if the difference in the attribute values of all the schemes under a certain attribute is smaller, the effect of the attribute on the decision is less strong. If an attribute makes a large difference in the attribute values of all the schemes, the attribute plays an important role in the decision. From the point of view of scheme ranking, if an attribute makes a larger deviation of the attribute values of all the schemes, a greater attribute weight should be given. In particular, if the attribute value of each scheme has no difference in a preferred evaluating indicator, it means that the evaluation index may not play a major role in the decision ranking of schemes, and its weight can be set as zero. According to the above considerations, the optimization model of attribute weight for decision making was established, and can be expressed as

$$
\left\{\begin{array}{lc}
\max : & f(w)=\sum_{j=1}^{M} \sum_{i=1}^{N} \sum_{k=1}^{N}\left|r_{i j}-r_{k j}\right| w_{j}^{*} \\
\text { where: } \quad w^{*}=\left(w_{1}^{*}, \quad w_{2}^{*}, \ldots, w_{m}^{*}\right) & \quad \sum_{j=1}^{M}\left(w_{j}^{*}\right)^{2}=1 \\
\text { s.t: } & \\
& 0 \leq w_{j}^{*} \leq 1 \quad i=1,2, \ldots, N, \quad j=1,2, \ldots, M, \quad k=1,2, \ldots, N
\end{array}\right.
$$

where $N$ is the number of schemes, $M$ is the number of evaluating indicators at the same level which belongs to one upper layer, $r_{i j}$ is the normalized attribute value, and $w_{j}^{*}$ is the weight satisfying unit constraint conditions.

The attribute weight can be calculated by the method of Lagrange multipliers, and the normalized attribute weight is

$$
w_{j}=\frac{\sum_{i=1}^{N} \sum_{k=1}^{N}\left|r_{i j}-r_{k j}\right|}{\sum_{j=1}^{M} \sum_{i=1}^{N} \sum_{k=1}^{N}\left|r_{i j}-r_{k j}\right|}
$$

Finally, the computed weight vector $w$ is the optimal weight obtained by the maximum deviation method. 


\subsection{Results}

The attribute values corresponding to the obtained optimal schemes of the outer dimensions of the throttling bar are listed in Table 2. The multiple attribute decision making method was used to calculate the specification values and the comprehensive attribute values of the scheme set $A$. In the attribute $\operatorname{set} u_{2}=\left(u_{21}, u_{22}, u_{23}\right)$, the attribute value of $u_{21}$ which indicates the effect of recoil resistance peaks is the interval numbers. Deterministic transformation of attribute values described by the interval numbers was conducted to obtain the sorting vector. As mentioned in Section 4.1, $u_{21}$ is the benefit type attribute, i.e. the larger the component, the better the result. Similar to $u_{21}$ are $u_{22}$ which indicates the effect of fullness of the recoil resistance curves and $u_{23}$ which indicates the effect of tolerance levels of the throttling bar outer diameters. They were normalized by using equation (9). On the other hand, $u_{23}$, indicating the effect of flatness of the recoil resistance curves, belongs to the cost type. It was normalized by using equation (8). All the specification values obtained by the normalization are given in Table 2 .

The weight vectors $\boldsymbol{w}_{2}=\left(w_{21}, w_{22}, w_{23}\right)$ and $\boldsymbol{w}=\left(w_{1}, w_{2}\right)$, corresponding to the attribute sets $u_{2}=\left(u_{21}, u_{22}, u_{23}\right)$ and $U=\left(u_{1}, u_{2}\right)$, respectively, were obtained by using the weight determination method based on maximum deviation. Here, $\boldsymbol{w}_{2}=(0.3507,0.3280,0.3213)$, and $\boldsymbol{w}=(0.4992,0.5008)$. The results of the five cases are listed in Table 2.

According to the comprehensive attribute values in Table 2, the schemes are sorted in the following order: $A_{3}>A_{4}>A_{2}>A_{5}>A_{1}$. From the point of views of recoil resistance and manufacturability, the optimization scheme of Case IT10 ranks as the best, the optimization scheme of Case IT11 ranks as the second, and then follows the Case IT9. It can be found that the weights of the recoil resistance and the manufacturability are almost equal. Generally, with the progress of processing technology, the weight of manufacturability will gradually be reduced. However, the results from the above method do not change with the improvement in the processability, and this may be felt to be a disadvantage of the presented method.

Table 2 The attribute values, specification values and comprehensive attribute values of scheme set $A$

\begin{tabular}{|c|c|c|c|c|c|c|}
\hline & Cases & $A_{1}$ & $A_{2}$ & $A_{3}$ & $A_{4}$ & $A_{5}$ \\
\hline \multirow[b]{2}{*}{$u_{21}$} & Attribute values & {$[168.9,178.8]$} & {$[169.2,179.6]$} & {$[169.9,182.8]$} & {$[170.4,182.7]$} & {$[174.9,192.5]$} \\
\hline & Specification values & 1 & 0.9196 & 0.6557 & 0.6263 & 0 \\
\hline \multirow[b]{2}{*}{$u_{22}$} & Attribute values & 0.9724 & 0.9705 & 0.9703 & 0.9648 & 0.9714 \\
\hline & Specification values & 1 & 0.75 & 0.7237 & 0 & 0.8684 \\
\hline \multirow[b]{2}{*}{$u_{23}$} & Attribute values & $3.009 \times 10^{5}$ & $3.037 \times 10^{5}$ & $3.098 \times 10^{5}$ & $3.043 \times 10^{5}$ & $3.611 \times 10^{5}$ \\
\hline & Specification values & 1 & 0.9535 & 0.8522 & 0.9435 & 0 \\
\hline \multirow[b]{2}{*}{$u_{11}$} & Attribute values & 8 & 9 & 10 & 11 & 12 \\
\hline & Specification values & 0.2848 & 0.5228 & 0.7411 & 0.8749 & 1 \\
\hline \multicolumn{2}{|c|}{$\begin{array}{c}\text { Comprehensive attribute } \\
\text { values }\end{array}$} & 0.4992 & 0.5371 & 0.5689 & 0.5417 & 0.5008 \\
\hline
\end{tabular}

\section{Conclusions}

This paper presents the multiple attribute decision making method with interval uncertainty for artillery recoil resistance. Some conclusions can be drawn from the study. The interval optimization design of the throttling bar outer diameters with different tolerance grades was carried out. The optimized recoil resistance curves are gentler and more uniform, with increased fullness. The multiple optimization schemes were sorted using the multiple attribute decision making method based on maximum deviation; the attribute values, the specification values and the comprehensive attribute values of the scheme set were calculated. 
The optimal design scheme of the throttling bar outer diameters, which considers simultaneously the manufacturability and the requirement of recoil resistance, was determined. The optimal interval of the recoil resistance peaks and the optimal recoil resistance curve with sufficient fullness and flatness were obtained. This study provides an approach to investigating optimization and decision making in the design of artillery components. In future work, the optimization and decision making in the design of artillery components with uncertainty firing loads taken into account will be carried out.

\section{Acknowledgement}

This work was supported by National Natural Science Foundation of China (Grant number: 51705253 and 11572158).

\section{REFERENCES}

[1] Zheng X J. Optimization design of a recoil mechanism. Dissertation of East China Institute of Technology, Nanjing, China, 1990.

[2] Zong S Z, Qian L F, Xu Y D, Dynamic coupling analysis and optimization of gun recoil mechanism. Acta Armamentarii, 2007, 28(3): 272-275.

[3] Zhou C, Gu K Q, Shao Y L. Optimal design of throttling bar in gun recoil mechanism. Journal of Gun Launch \& Control, 2012 (4): 68-70.

[4] Song J, He Y, Zhao W, Tao Q G. Optimization structural design and analysis on recoil mechanism of howitzer mortar. Journal of Sichuan Ordnance, 2013, 34(7): 22-24.

[5] Liu Q H, Xu Y D, Yang L. Parameterization design and optimization of throttling bar recoil mechanism. Journal of Gun Launch \& Control, 2013 (4): 49-53.

[6] Li Q, Zheng J G, Gao S Z. Optimal design of firing stability and artillery recoil resistance R. Journal of Gun Launch \& Control, 1997 (1): 34-39.

[7] Cui K B, Qin J Q, Di C C, Zhang Y J. Research on artillery structural parameter optimization based on ADAMS and CPSO algorithm. Chinese Journal of Engineering Design, 2012, 19(4): 278-282.

[8] Guha A. Continuous process control using neural networks. Journal of Intelligent Manufacturing, 1992, 3(4): 217-228. https://doi.org/10.1007/BF01473899

[9] Jiang C. Theories and algorithms of uncertain optimization based on interval. Dissertation of Hunan University, Changsha, China, 2008.

[10] Li R, Yang G L, Sun Q Z, Xiao H. Optimization on interval uncertainty of artillery recoil resistance. Journal of Ballistics, 2017, 29(2): 78-84.

[11] Jiang C, Xie H C, Zhang Z G, Han X. A new interval optimization method considering tolerance design. Engineering Optimization, 2015, 47(12): 1637-1650. https://doi.org/10.1080/0305215X.2014.982632

[12] Xu Z S, Da Q L, Possibility degree method for ranking interval numbers and its application. Journal of Systems Engineering, 2003, 18(1): 67-70.

[13] Kenny Q Y, Li W, Sudjianto A. Algorithmic construction of optimal symmetric Latin hypercube designs. Journal of Statistical Planning and Inference, 2000, 90(1): 145-159. https://doi.org/10.1016/S03783758(00)00105-1

[14] Li R. Interval optimization and neural network evaluation for error schemes of key artillery parameters. Dissertation of Nanjing University of Science and Technology, Nanjing, China, 2017.

[15] Altuntas S, Dereli T. An evaluation index system for prediction of technology commercialization of investment projects. Journal of Intelligent \& Fuzzy Systems, 2012, 23(6): 327-343. https://doi.org/10.3233/IFS-2012-0524

[16] Ma B, Najafi M, Shen H, Wu L. Risk evaluation for maxi horizontal directional drilling crossing projects. Journal of Pipeline Systems Engineering and Practice, 2010, 1(2): 91-97. https://doi.org/10.1061/(ASCE)PS.1949-1204.0000046

[17] Tamiz M, Jones D, Romero C. Goal programming for decision making: An overview of the current stateof-the-art. European Journal of Operational Research, 1998, 111(3): 569-581.

https://doi.org/10.1016/S0377-2217(97)00317-2 
[18] Das S, Dutta B, Guha D. Weight computation of criteria in a decision-making problem by knowledge measure with intuitionistic fuzzy set and interval-valued intuitionistic fuzzy set. Soft Computing, 2016, 20(9): 3421-3442. https://doi.org/10.1007/s00500-015-1813-3

[19] Hao Z N, Xu Z S, Zhao H, Fujita H. A dynamic weight determination approach based on the intuitionistic fuzzy Bayesian network and its application to emergency decision making. IEEE Transactions on Fuzzy Systems, 2017, 26(4): 1893-1907. https://doi.org/10.1109/TFUZZ.2017.2755001

Submitted: $\quad 27.02 .2019$

Accepted: $\quad 06.4 .2020$
Rong Li

School of Mechanical Engineering, Nanjing University of Science and Technology, Nanjing, P.R. China The 724 Research Institute of China Shipbuilding Industry Corporation, Nanjing, P.R. China

Quanzhao Sun*

School of Mechanical Engineering, Nanjing University of Science and Technology, Nanjing, P.R. China Inner Mongolia North Heavy Industries Group Corp. Ltd, Baotou, P.R. China Jie Zhang Yanming Song Inner Mongolia North Heavy Industries Group Corp. Ltd, Baotou, P.R. China Guolai Yang

Baochun Lu

School of Mechanical Engineering, Nanjing University of Science and Technology, Nanjing, P.R. China *Corresponding author, sunquanzhao@njust.edu.cn 